\title{
On reciprocally weighted partitions
}

by

D. E. Lehmor (Berkeley, Calif.)

Dedicated to the memory of Wactaw Sierpinstit

Introduction. Let $n$ be a positive integer and let

$$
P: a_{1}+a_{2}+a_{3}+\ldots=n \quad\left(0<a_{1} \leqslant a_{2} \leqslant a_{3} \leqslant \ldots\right)
$$

be any partition of $n$ into positive integer parts. We assign to this partition the weight

$$
w(P)=\left(a_{1} a_{2} a_{3} \ldots\right)^{-1} .
$$

If the parts $a_{i}$ are restricted to a set $S$ of positive integers and if we add the weights of all the partitions of $n$ into parts talien from $S$ we obtain

$$
W_{n}(S)=\sum w(P)
$$

a rational number we call informally the weighted number of partitions of $n$ into parts belonging to $S$. If we require that the parts in (1) be distinct we obtain, in lieu of (2) a smaller sum, which we denote by $W_{n}^{*}(S)$, called the weighted number of partitions of $n$ into distinct parts belonging to $S$. For example if $S$ is unrestricted and if $n=6$ we have eleven partitions.

\begin{tabular}{rrrr}
\multicolumn{1}{c}{$P$} & $w(P)$ & \multicolumn{1}{c}{$P$} & $w(P)$ \\
\hline 6 & $1 / 6$ & $1+1+1+3$ & $1 / 3$ \\
$1+5$ & $1 / 5$ & $2+2+2$ & $1 / 8$ \\
$2+4$ & $1 / 8$ & $1+1+2+2$ & $1 / 4$ \\
$1+1+4$ & $1 / 4$ & $1+1+1+1+2$ & $1 / 2$ \\
$3+3$ & $1 / 9$ & $1+1+1+1+1+1$ & 1 \\
$1+2+3$ & $1 / 6$ & &
\end{tabular}

Hence.

$$
W_{6}(S)=\frac{581}{180} \quad \text { and } W_{6}^{*}(S)=\frac{1}{6}+\frac{1}{5}+\frac{1}{8}+\frac{1}{6}=\frac{79}{120}
$$


If we multiply $W_{n}(S)$ and $W_{n}^{*}(S)$, by $n$ ! we obtain two non-negative integers

$$
A_{n}(S)=n ! W_{n}\left(S^{\prime}\right), \quad A_{n}^{*}\left(S^{\prime}\right)=n ! W_{n}^{*}\left(S^{\prime}\right) .
$$

That these are indeed integers follows from the fact that evcry term $w(P)$ becomes an integer when multiplied by $n !$. In fact even

$$
\frac{n !}{a_{1} ! a_{2} ! \ldots}=\frac{n ! w(P)}{\left(a_{1}-1\right) !\left(a_{2}-1\right) ! \ldots}
$$

is an integer since it is a multinomial cocfficient.

We adopt the convention.

$$
A_{0}(S)=A_{0}^{*}(S)=1
$$

Generating functions. If we expand the following products into power series we see that $A$ and $A^{*}$ are generated by

$$
\begin{aligned}
& F(x)=H^{\prime}(x, S)=\prod_{m \in S}\left(1-x^{n} / m\right)^{-1}=\sum_{n=0}^{\infty} A_{n}(S) x^{n} / n ! \\
& F^{*}(x)=F^{*}(x, S)=\prod_{m \in S}\left(1+x^{m} / m\right)=\sum_{n=0}^{\infty} A_{n}^{*}(S) x^{n} / n !
\end{aligned}
$$

The integers $A_{n}$ and $A_{n}^{*}$ may be computed recursively thus avoiding the generation of all the corresponding partitions of $n$ in terms of which they are defined. To this effect we have

Tweorem 1. Define $\Gamma_{n}(S)=\Gamma_{n}$ and $\Gamma_{n}^{*}(S)=\Gamma_{n}^{*}$ by

$$
\Gamma_{0}=\Gamma_{0}^{*}=0, \quad \Gamma_{n}=n ! \sum_{\substack{\delta, n \\ \delta \in S}} \delta^{1-n / \delta}, \quad \Gamma_{n}^{*}=n ! \sum_{\substack{\delta, n \\ \delta \in S}}(-\delta)^{1-n / \delta},
$$

where the sums extend over all those divisors of $n$ which belong to $S$.

Then, symbotically,

$$
n A_{n}=(A+\Gamma)^{n} \quad \text { and } \quad n A_{n}^{*}=\left(A^{*}+\Gamma^{*}\right)^{n} .
$$

In other words,

$$
n A_{n}=\sum_{k=1}^{n} A_{n-k}\left(\begin{array}{l}
n \\
k
\end{array}\right) \Gamma_{k}, \quad n A_{n}^{*}=\sum_{k=1}^{n} A_{n-1 k}^{*}\left(\begin{array}{l}
n \\
b_{k}
\end{array}\right) I_{k}^{*} .
$$

Pro of. To prove the first of these conclusions we take the logarithmic derivative of $F(x)$, thus

$$
\begin{aligned}
\frac{x E^{\prime}(x)}{\bar{F}(x)} & =\sum_{m \in S} \frac{x^{m}}{1-x^{m} / m}=\sum_{m \in S} x^{m} \sum_{n=0}^{\infty} x^{m n} m^{-n}=\sum_{m \in S} \sum_{r=1}^{\infty} x^{m n r} m^{1-r} \\
& =\sum_{k=1}^{\infty} x^{k} \sum_{\substack{m r=l_{k} \\
m \in S}} m^{1-r}=\sum_{k=1}^{\infty} x^{k} \Gamma_{k} / k !
\end{aligned}
$$

Multiplying by $F(x)$ and identifying coefficients of $x^{n}$ on both sides gives

$$
n A_{n} / n !=\sum_{k=0}^{n} T_{k} A_{n-k} /[(k !)(n-k) !]
$$

or

$$
n A_{n}=\sum_{k_{k}=0}^{n} \Gamma_{k}\left(\begin{array}{l}
n \\
k
\end{array}\right) A_{n-l_{k}}=(A+T)^{n}
$$

The formula $n A_{n}^{*}=\left(A^{*}+\Gamma^{*}\right)^{n}$ is proved in the same way using $F^{*}(x)$ CoROLLARX. $\Gamma_{k}$ and $\Gamma_{k}^{*}$ are integers.

Proof. By Theorem 1

$$
\Gamma_{n}=n A_{n}-\sum_{k=1}^{n-1} \Gamma_{l k}\left(\begin{array}{l}
n \\
l k
\end{array}\right) A_{n-k k} .
$$

Hence, by complete induction, $\Gamma_{n}$ is an integer since its predecessors are. Similar reasoning applies to $\Gamma_{k}^{*}$.

Simple limit theorems. Table $I$ gives the first ten values of the six functions $A, A^{*}, T, T^{*}, W, W^{*}$ in the unrestricted case, i.e., when $S$ is the set of all positive integers.

\begin{tabular}{rrrrrrrr} 
& \multicolumn{7}{c}{ Table I } \\
$n$ & \multicolumn{1}{c}{$\Gamma_{n}$} & \multicolumn{1}{c}{$A_{n}$} & $W_{n}$ & $W_{n} / n$ & \multicolumn{1}{c}{$\Gamma_{n}^{*}$} & \multicolumn{1}{c}{$A_{n}^{*}$} & \multicolumn{1}{c}{$W_{n}^{*}$} \\
\hline 1 & 1 & 1 & 1.00000 & 1.00000 & 1 & 1 & 1.00000 \\
2 & 4 & 3 & 1.50000 & .75000 & 0 & 1 & .50000 \\
3 & 12 & 11 & 1.83333 & .61111 & 12 & 5 & .83333 \\
4 & 60 & 56 & 2.33333 & .58333 & -12 & 14 & .58333 \\
5 & 240 & 324 & 2.70000 & .54000 & 240 & 74 & .61667 \\
6 & 1860 & 2324 & 3.22778 & .53796 & -60 & 474 & .65833 \\
7 & 10080 & 18332 & 3.63730 & .51961 & 10080 & 3114 & .61786 \\
8 & 95760 & 167544 & 4.15536 & .51942 & -15120 & 24240 & .60119 \\
9 & 766080 & 1674264 & 4.61382 & .51265 & 766080 & 219456 & .60476 \\
10 & 8210160 & 18615432 & 5.12991 & .51299 & -498960 & 2231280 & .61488 \\
& & & & & & &
\end{tabular}

Inspection of this small table of $W_{n}^{*}$ leards one to guess that $W_{n}^{*}$ tends to some limit. This is confimed by

THEOREM 2. The weighted number of partitions of $n$ into distinct parts tends to $e^{-\gamma}$ as $n \rightarrow \infty$. That is

$$
\lim _{n \rightarrow \infty} W_{n}^{*}=e^{-\gamma}=.56145948 \ldots,
$$

where $\gamma$ is Euler's constant. 
Proof. With $S$ the set of all positive integers let

$$
G^{*}(x)=(1-x) F^{*}(x)=\sum_{n=0}^{\infty} b_{n} x^{n}=1-\frac{1}{2} x^{2}+\frac{1}{3} x^{3}-\frac{1}{4} x^{4}+1-\frac{1}{30} x^{5} \ldots
$$

so that

$$
W_{n}^{*}=A_{n} \mid n !=\sum_{k=0}^{n} b_{k} \text {. }
$$

Then, by (4),

$$
\begin{aligned}
\log G^{*}(x) & =\log (1-x)+\sum_{n=1}^{\infty} \log \left(1+x^{n} / n\right) \\
& =-\sum_{n=1}^{\infty} \frac{x^{n}}{n}+\sum_{n=1}^{\infty} \sum_{m=1}^{\infty} \frac{(-1)^{m-1}}{m n^{n}} x^{m n}=-\sum_{n=1}^{\infty} \sum_{n=2}^{\infty} \frac{(-1)^{m}}{m n^{m}} x^{m n} .
\end{aligned}
$$

This series converges for $x=1$ and

$$
\begin{aligned}
-\log G^{*}(1) & =\sum_{n=1}^{\infty} \sum_{m=2}^{\infty} \frac{(-1)^{m}}{m n^{m}}=\lim _{N \rightarrow \infty}\left\{\sum_{n=1}^{N}\left(\log \left(1+\frac{1}{n}\right)-\frac{1}{n}\right)\right\} \\
& =\lim _{N \rightarrow \infty}\left\{\log (1+N)-\sum_{n=1}^{N} 1 / n\right\}=\gamma .
\end{aligned}
$$

Hence

$$
e^{-\gamma}=G^{*}(1)=\lim _{N \rightarrow \infty} \sum_{n=0}^{N} b_{n}=\lim _{N \rightarrow \infty} W_{N}^{*}
$$

which proves the theorem.

Further inspection of Table I suggests that $W_{n} / n$ also tends to a limit. This is confirmed by

THEOREM 3. The weighted number of unrestricted partitions of $n$ is asymptotic to $e^{-\gamma} n$. That is

$$
\lim _{n \rightarrow \infty} W_{n} \mid n=e^{-\gamma}
$$

Before proving this theorem it is convenient to prove

Thoorem 4. The weighted number of partitions of $n$ into parts $>1$ tends to $e^{-\gamma}$ as $n \rightarrow \infty$.

Proof. Let $S_{1}$ now be the set of all integers $\geqslant 2$ and let $F(x)$ mean $F\left(x, S_{1}\right)$, then

$$
G(x)=(1-x) F(x)=\sum c_{n} x^{n}=\frac{x^{2}}{2}-\frac{x^{3}}{6}+\cdots
$$

so that

$$
\mathbb{W}_{n}\left(S_{1}\right)=A_{n}\left(S_{1}\right) / n !=\sum_{k=0}^{n} c_{k}
$$

Then by (3)

$$
\begin{aligned}
\log G(x) & =\log (1-x)-\sum_{n=2}^{\infty} \log \left(1-x^{n} / n\right) \\
& =\sum_{n=2}^{\infty} \sum_{m=1}^{\infty} \frac{x^{m n}}{m n^{m}}-\sum_{n=1}^{\infty} \frac{x^{n}}{n}=-x+\sum_{n=2}^{\infty} \sum_{m=2}^{\infty} \frac{x^{m n}}{m n^{m}}
\end{aligned}
$$

a series that converges at $x=1$.

Fence

$$
\begin{aligned}
\log Q(1) & =-1+\lim _{N \rightarrow \infty} \sum_{n=2}^{N} \sum_{m=2}^{\infty} \frac{1}{m n^{m}}=-1+\lim _{N \rightarrow \infty} \sum_{n=2}^{N}\left\{\sum_{m=1}^{\infty} \frac{\left(n^{-1}\right)^{m}}{m}-\frac{1}{n}\right\} \\
& =\lim _{N \rightarrow \infty}\left\{\sum_{n=2}^{N} \log \left(\frac{n}{n-1}\right)-\sum_{n=1}^{N} \frac{1}{n}\right\}=\lim _{N \rightarrow \infty}\left\{\log N-\sum_{n=1}^{N} \frac{1}{n}\right\}=-\gamma .
\end{aligned}
$$

Fence

$$
e^{-\gamma}=G(1)=\lim _{N \rightarrow \infty} \sum_{n=0}^{N} c_{n}=\lim _{N \rightarrow \infty} W_{N}\left(S_{1}\right),
$$

which proves Theorem 4.

To prove Theorem 3 we observe that unrestricted partitions of $n$ are of two types:

(a) Those involving the part 1.

(b) Those with anl parts $>1$.

Those of type (a) correspond uniquely to an unrestricted partition of $n-1$ simply by supressing one unit part. These two partitions have the same weight. Hence

Therefore

$$
W_{n}-W_{n-1}=W_{n}\left(S_{1}\right)
$$

$$
\lim _{N \rightarrow \infty} \frac{W_{N}}{N}=\lim _{N \rightarrow \infty} \frac{1}{N} \sum_{n=1}^{N}\left(W_{n}-W_{n-1}\right)=\lim _{N \rightarrow \infty} \frac{1}{N} \sum_{n=1}^{N} W_{n}\left(S_{1}\right) .
$$

By Theorem 4 this average must tend to $e^{-\gamma}$. This proves Theorem 3 .

Arithmetical progressions. In order to treat partitions whose parts lie in an arithmetical progression we need two lemmas.

LEMma 1. Let $c_{n} \geqslant 0$ and let $f(x)=\sum_{n=0} c_{n} x^{n}$ be such that for some $\lambda>0$

$$
\lim _{x \rightarrow 1-0}(1-x)^{2} f(x)=0
$$

Then

$$
\lim _{n \rightarrow \infty} n^{-\lambda}\left(c_{0}+c_{1}+\ldots+c_{n}\right)=C / \Gamma(\lambda)
$$


Proof. For $\lambda=1$ this is a Tauberian theorem of Haxdy ([1], Theorem 96, p. 155). For a general $\lambda$ we need to observe that as $x \rightarrow 1-0$

$$
f(x) \sim O(1-x)^{-\lambda}=C \sum_{n=0}^{\infty}\left(\begin{array}{c}
n+\lambda-1 \\
n
\end{array}\right) x^{n}
$$

Furthermore

$$
\begin{aligned}
\left(\begin{array}{c}
n+\lambda-1 \\
n
\end{array}\right) & =\frac{\Gamma(n+\lambda)}{\Gamma(n+1) \Gamma(\lambda)} \\
& =\frac{1}{\Gamma(\lambda)}(n+\lambda-1)(n+\lambda-2) \ldots(n+1) \sim n^{\lambda-1} / \Gamma(\lambda)
\end{aligned}
$$

With these modifications the proof goes through.

LEMTMA 2. If $a>0$,

$$
\lim _{x \rightarrow 1-0}(1-x)^{1 / a} \prod_{n=1}^{\infty}\left(1-\frac{x^{a n+b}}{a n+b}\right)^{-1}=a^{-1 / a} e^{-\gamma / a}\left\{\Gamma\left(1+\frac{b-1}{a}\right) / \Gamma\left(1+\frac{b}{a}\right)\right\} .
$$

Proof. Let

$$
Q(x)=\left(1-x^{a}\right)^{-1 / a} \prod_{n=1}^{\infty}\left(1-\frac{x^{a n+b}}{a n+b}\right) .
$$

For typographical simplicity set

\section{then}

$$
y=x^{\alpha}, \quad a=1 / a, \quad b a=c,
$$

$\left(1-x^{a}\right)^{1 / a}=(1-y)^{\alpha}=\exp \{\alpha \log (1-y)\}=\exp \left(\sum_{n=1}^{\infty} \frac{-\alpha y^{n}}{n}\right)=\prod_{n=m i}^{\infty} e^{-\alpha y^{n / n}}$.

\section{Hence}

$$
\begin{aligned}
G(x) & =\prod_{n=1}^{\infty}\left\{\left(1-\frac{\alpha y^{n+c}}{n+c}\right) e^{\alpha y^{n} / n}\right\} \\
& =e^{\alpha y}\left(1-\frac{\alpha y^{c+1}}{c+1}\right) \prod_{n=1}^{\infty}\left\{\left(1-\frac{\alpha y^{n+c+1}}{n+c+1}\right) e^{\alpha y^{n+1} /(n+1)}\right\} \\
& =e^{\alpha y} \frac{c+1-\alpha y^{c+1}}{c+1} \prod_{n=1}^{\infty}\left\{\left(1+\frac{e+1-\alpha y^{n+c+1+1}}{n}\right) e^{-\left(c+1-\alpha y^{n+1+1+c) / n}\right\} \times}\right. \\
& \times\left\{\prod_{n=1}^{\infty}\left\{\left(1+\frac{c+1}{n}\right) e^{-(c+1) / n}\right\}\right\}^{-1}\left\{\prod_{n=1}^{\infty} \exp \left\{\alpha\left[\frac{y^{c}}{n}-\frac{1}{n+1}\right] y^{n+1}\right\}\right\}^{-1}
\end{aligned}
$$

Since

$$
\Gamma(z)=z^{-1} e^{-y z} \prod_{n=1}^{\infty}\left\{\left(1+\frac{z}{n}\right)^{-1} e^{z / n}\right\}
$$

we have

$$
\begin{aligned}
G(x)=\Gamma(1+c) e^{y(1+c)}\left(c+1-\alpha y^{c+1}\right) \times & \\
& \times \exp \left\{\alpha\left[y+\sum_{n=1}^{\infty} \frac{y^{n+1}}{n+1}-y^{c+1} \sum_{n=1}^{\infty} \frac{y^{n}}{n}\right]\right\} P(y)
\end{aligned}
$$

where

$$
P(y)=\prod_{n=1}^{\infty}\left\{\left(1+\frac{c+1-\alpha y^{n+c+1}}{n}\right) e^{-\left(c+1-\alpha y^{n+c+1}\right) / n}\right\} .
$$

The expression inside the square brackets is simply

$$
-\left(1-y^{c+1}\right) \log (1-y)
$$

and this vanishes as $y$ tends to 1 .

Next we consider

$$
\lim _{y \rightarrow 1-0} P(y)
$$

The logarithm of the $n$th factor is

$$
\begin{aligned}
T_{n} & =-\frac{c+1-\alpha y^{n+c+1}}{n}+\log \left(1+\frac{c+1-\alpha y^{n+c+1}}{n}\right) \\
& =\sum_{m=2}^{\infty} \frac{(-1)^{m+1}\left(c+1-\alpha y^{c+1+n}\right)^{m}}{m n^{m}} .
\end{aligned}
$$

Let

and lot

$$
v=o+1-\alpha
$$

Then for $n>N$

$$
N>2 \nu
$$

$$
\left|T_{n}\right|<\frac{\nu^{2}}{n^{2}}\left(1+\frac{|\nu|}{n}+\frac{|\nu|^{2}}{n^{2}}+\ldots\right)<\frac{N^{2}}{4 n^{2}} \sum_{\lambda=0}^{\infty} 2^{-\lambda}=\frac{1}{2}\left(\frac{N}{n}\right)^{2} .
$$

Since

$$
\sum_{n>N} N^{2} / n^{2}
$$


converges it follows that $\log P(y)$ is analytic for $|y| \leqslant 1$, and hence $P(y)$ tends to

$$
P(1)=\prod_{n=1}^{\infty}\left\{\left(1+\frac{v}{n}\right) e^{-v / n}\right\}=e^{-\nu \nu} /\{\nu T(\nu)\}
$$

Hence

$$
\lim _{x \rightarrow 1-0} G(x)=\Gamma(c+1) e^{\gamma(c+1)} p P(1)=e^{(x \gamma} T^{\gamma}(c+1) / \Gamma(c+1-\alpha)
$$

It remains to observe that

$$
\lim _{x \rightarrow 1} \frac{(1-x)^{1 / a}}{\left(1-x^{a}\right)^{1 / a}}=a^{-1 / a}
$$

and to restore the variables $a$ and $b$ in terms of $\alpha$ and $c$ to obtain the theorem.

THEOREM 5. Let $S_{w}$ denote the set of all odd numbers. Then for the weighted number $W_{n}\left(S_{w}\right)$ of partitions of $n$ into odd parts we have

$$
W_{n}\left(S_{w}\right) \sim \frac{2}{\pi} \sqrt{2 e^{-\gamma} n}=.674: 6124 \sqrt{n}
$$

Proof. In Lemma 2 we put $a=2$ and $b=1$, we find

$$
\lim _{x \rightarrow 1-0}(1-x)^{1 / 2} \prod_{n=1}^{\infty}\left(1-\frac{x^{2 n+1}}{2 n+1}\right)^{-1}=2^{-1 / 2} e^{-\gamma / 2} / \Gamma(3 / 2)=\sqrt{\frac{2}{e^{y} \pi}}
$$

Since the first factor $(1-x)^{-1}$ of the generator of $W_{n}\left(S_{w}\right)$ is missing from the above product we have

$$
\lim _{x \rightarrow 1 \rightarrow 0}(1-\infty)^{3 / 2} \sum_{n *=0}^{\infty} W_{n}\left(S_{w}\right) x^{n}=\sqrt{\frac{2}{e^{\prime} \pi}}
$$

By Lemma 1. therefore

$$
\sum_{r=0}^{n} W_{r}\left(S_{w}\right) \sim n^{3 / 2} \sqrt{\frac{2}{e^{\gamma} \pi}} / \Gamma(3 / 2)=n^{3 / 2} \sqrt{8 e^{-m \nu}} / \pi .
$$

Since $W_{n}\left(S_{w}\right)$ is an increasing function of $n$

$$
\lim _{n \rightarrow \infty} W_{n}\left(S_{w}\right) n^{-1 / 2}=\lim _{n \rightarrow \infty} n^{-3 / 2} \sum_{r=0}^{n} W_{r}\left(S_{w}\right)=\sqrt{8 e^{-\gamma / 1}} / \pi
$$

THEonem 6. Let $S_{e}$ denote the set of all even numbers. Then for the weighted number $W_{n}\left(S_{e}\right)$ of partitions of $n$ into even parts we have $W_{n}\left(S_{e}\right)=0$ if $n$ is odd, while

$$
W_{n}\left(S_{e}\right) \sim \sqrt{2 e^{-\gamma}} / \sqrt{n}=1.0596787 / \sqrt{n}
$$

if $n$ is even.

Proof. If we put $a=2$ and $b=0$ in Lemma 2 we find

$$
\lim _{x \rightarrow 1-0}(1-x)^{1 / 2} \prod_{n=1}^{\infty}\left(1-\frac{x^{2 n}}{2 n}\right)^{-1}=2^{-1 / 2} e^{-\gamma / 2} \Gamma(1 / 2)=\sqrt{\frac{\pi}{2 e^{\gamma}}} .
$$

Hence by Lemma 1 we have

$$
\sum_{r=0}^{n} W_{r}\left(S_{e}\right) \sim n^{1 / 2} \sqrt{\frac{\pi}{2 e^{\gamma}}} / \Gamma(1 / 2)=n^{1 / 2} / \sqrt{2 e^{\gamma}} .
$$

$$
\frac{2}{n} \sum_{r=0}^{n} \sqrt{n} \mathrm{~W}_{r}\left(S_{e}\right) \sim \sqrt{2 e^{-\eta}}
$$

Only half the terms of this sum are non-zero. So we have an average of the even ordered terms.

Hence when $n$ is even

$$
\sqrt{n} W_{n}\left(S_{e}\right) \rightarrow \sqrt{2 e^{-\gamma}} \quad \text { as } \quad n \rightarrow \infty .
$$

For the general arithmetic progression we have

THEOREM 7. Let $a>1$ and denote by $S_{a, b}$ the set of all positive integers congruent to $b$ moduto a with $0 \leqslant b<a$. Then as $n \rightarrow \infty$

$$
\begin{gathered}
W_{n}\left(S_{a, 0}\right) \sim\left\{a^{I-I / a} e^{-\gamma / a} \Gamma\left(1-\frac{1}{a}\right) \Gamma\left(\frac{1}{a}\right)\right\} n^{1 / a-1} \text { if } a \mid n, \\
W_{n}\left(S_{a, 0}\right)=0 . \quad \text { if } \quad a \nmid n \\
W_{n}\left(S_{a, 1}^{\prime}\right) \sim\left\{a^{-1 / a} e^{-\gamma / a} /\left[\Gamma\left(1+\frac{1}{a}\right)\right]^{2}\right\} n^{1 / a} .
\end{gathered}
$$

If $b \neq 0$ and $b \neq 1$,

$$
W_{n}\left(\Phi_{a, b}\right) \sim\left\{a^{1-1 / a} e^{-\gamma / a} \Gamma\left(\frac{b-x}{a}\right) \Gamma\left(\frac{b}{a}\right) / \Gamma\left(\frac{1}{a}\right)\right\} n^{1 / a-1} .
$$

The proof follows the lines of the proofs of Theorems 5 and 6 .

Numerical values. In conclusion we give in Table IT brief numerical evidence that the various limits discussed above as approached at rather 
leisurely rates. The five functions of the following table refer to partitions jnto distinct parts, parts $>1$, unrestricted parts, odd parts and even parts respectively.

Tabie II

\begin{tabular}{lllllc}
$n$ & $W_{n}^{*}$ & $W_{n}\left(S_{1}\right)$ & $W_{n} / n$ & $W_{n}\left(S_{w}\right) / \sqrt{n}$ & $\sqrt{n} W_{n}\left(S_{n}\right)$ \\
\hline 100 & .566786 & .555790 & .542158 & .609193 & 1.072995 \\
101 & .566726 & .555423 & .542289 & .669292 & 0 \\
102 & $.56669 \mathrm{~J}$ & .555910 & .542423 & .669277 & 1.071161 \\
103 & .566634 & .555546 & .542550 & .669309 & 0 \\
104 & .566584 & .55601 .1 & .542680 & .609432 & 1.072575 \\
Limit & .561459 & .561459 & .561459 & .674612 & 1.05968
\end{tabular}

The slight irregularities in these frunctions are not due to inaccuracy. They reflect the existence of an asymptotic, or possibly convergent, series for each entry.

\section{Reference}

[1] G. H. Hardy, Divergent Series, Oxford 1949 .

Received on $17.8,1971$

\section{Some diophantine equations solvable by identities}

by

A. Matrowskt (Warszawa)

Dedicated to the memory of my teacher Wactaw Sierpinstai

1. W. Sierpinski in many of his papers investigated the triangular numbers $t_{n}=\frac{1}{2} n(n+1)$ and tetrahedral numbers $T_{n}=\frac{1}{6} n(n+1)(n+2)$.

From the identity given by A. Gérardin. [1] we get immediately the following identity

$$
\left(27 n^{6}\right)^{2}-1=\left(9 n^{4}-3 n\right)^{3}+\left(9 n^{3}-1\right)^{3}=\left(9 n^{4}+3 n\right)^{3}-\left(9 n^{3}+1\right)^{3} .
$$

With $n$ odd and positive the last identity provides infinitely many integer solutions of the equation

$$
(2 x+1)^{2}-1=(2 y)^{3}+(2 z)^{3}=(2 u)^{3}-(2 v)^{3}
$$

which is equivalent to

$$
t_{x}=y^{3}+z^{3}=u u^{3}-v^{3} .
$$

Thus there exist infinitely many triangular numbers which are simultaneously representable as sums and differences of two positive cubes.

We have the identity $3 a T_{a-1}=t_{a^{2}-1}$. Since there exist infinitely many tetrahedral numbers divisible by $3: T_{m}=3 a$ we infer that there exist infinitely many triangular numbers which are products of two tetrahedral numbers $>1$.

2. The numbers $x=6^{2} p r^{2} n^{3}+6^{6} p^{4} r^{5} n^{2}, \quad y=6^{2} p r^{2} n^{3}-6^{6} p^{4} r^{5} n^{9}$, $z=6^{5} p^{3} r^{4} n^{7}$ satisfy the equation

$$
p\left(x^{3}+y^{3}-z^{3}\right)=r(x-y) .
$$

This answers a question posed by A. Oppenheim in [3].

3. L. J. Mordell [2] investigated the equation $z^{2}=a x^{3}+b y^{3}+c$. It may be noticed that the equation

$$
z^{2}=a x^{2 k+1}+b y^{2 k+1}+c
$$

